\title{
Mesenchymal stromal cells and macrophages in sepsis: new insights
}

\author{
Maya E. Kotas ${ }^{1}$ and Michael A. Matthay ${ }^{2}$ \\ Affiliations: ${ }^{1}$ Dept of Medicine, University of California, San Francisco, CA, USA. ${ }^{2}$ Depts of Medicine and \\ Anesthesia and the Cardiovascular Research Institute, University of California, San Francisco, CA, USA.
}

Correspondence: Michael A. Matthay, 505 Parnassus Avenue, M-917, University of California, San Francisco, CA 94143, USA. E-mail: Michael.matthayqucsf.edu

@ERSpublications

A better understanding of the effects of mesenchymal stromal cells on the function of macrophages may open up new therapeutic opportunities for treating sepsis http://ow.ly/8eRx30j28iH

Cite this article as: Kotas ME, Matthay MA. Mesenchymal stromal cells and macrophages in sepsis: new insights. Eur Respir J 2018; 51: 1800510 [https://doi.org/10.1183/13993003.00510-2018].

Sepsis is a syndrome of acute organ dysfunction caused by the physiologic and pathophysiologic response to infection [1-3]. Although the specific clinical definition of sepsis continues to evolve, the entity has been recognised in some form or other for thousands of years. Yet, distressingly, over 30 million cases of sepsis still occur globally each year, resulting in over 5 million deaths, and inestimable disability $[1,3]$. The pathophysiology of sepsis is perplexing, as some features indicate an overly exuberant inflammatory response, while others reveal relative immunosuppression $[2,4,5]$. These processes may be concurrent, or may be separated by space (i.e. tissues) and time. Immune dysfunction likely triggers the coagulation abnormalities, endothelial and epithelial barrier disruption, and altered vasoactivity that culminate in multiorgan dysfunction. However, the specific cellular and molecular pathways that are responsible are incompletely understood. These uncertainties surrounding the pathophysiology of sepsis are highlighted by the lack of targeted therapies. With the exception of antibiotics, essentially all of the interventions for sepsis are supportive, rather than directed towards a specific pathophysiology. Thus, targeted therapies to reverse the tissue pathology that occurs during sepsis are needed.

Mesenchymal stromal cells (MSC) are mesodermal cells capable of in vitro differentiation into osteocytes, adipocytes and chondrocytes, and are found in multiple tissues including the bone marrow, adipose, lung, heart, muscle and fetal tissues $[6,7]$. Over little more than a decade, numerous immunomodulatory and tissue reparative functions have been ascribed to MSCs [6-8]. As a result, there has been substantial interest in their potential for various clinical applications, including sepsis. In fact, numerous preclinical studies have successfully used MSCs to improve outcomes in animal models of sepsis and organ injury [9], and clinical studies to test their potential are ongoing $[6,9]$. Several studies have shown a reduction in inflammatory cytokines with administration of MSCs, as well as the induction of IL-10 through interaction with macrophages $[7,10-13]$. However, MSCs also appear to augment macrophage-mediated phagocytosis and bacterial killing [7, 10,13-16], a feature seemingly at odds with their anti-inflammatory properties. Therefore, a comprehensive understanding of MSC-macrophage relationships remains elusive.

Macrophages are known to exhibit various functional phenotypes, dependent on their local microenvironment. These states have been described as polarisation because they are reversible, in contrast to differentiation, which is conceptualised (perhaps over-simplistically) as fixed. Although there are now many such phenotypes that have been described for macrophages, presumably because each tissue microenvironment is so distinct, two well-described macrophage phenotypes are termed M1 and M2 after their association with Th1 and Th2 T-cell effector responses. The M1 phenotype, or classically activated 
macrophages, is generated when monocyte-derived macrophages are stimulated with interferon (IFN)- $\gamma$ or bacterial lipopolysaccharide. These cells produce abundant inflammatory cytokines such as tumour necrosis factor (TNF)- $\alpha$ and interleukin (IL)-12, and reactive oxygen and nitrogen species aimed at microbial killing. M2 macrophages, generated by IL-4/-13 stimulation, express a variety of lectins, scavenger receptors and secreted proteins useful for fungal and helminthic control, as well as enzymes that aid in collagen production and tissue repair [17, 18]. Both populations produce the anti-inflammatory cytokine IL-10, though to different degrees [17]. Based on this understanding of macrophage biology, it has been unclear how MSCs could suppress M1-like inflammatory cytokine expression and augment M2-like differentiation while simultaneously enhancing the oxidative burst.

In this issue of the European Respiratory Journal, RABANi et al. [19] investigated these seemingly discordant prior findings to improve our understanding of MSC effects on macrophages. They found that when co-cultured with human MSCs in vitro, human peripheral blood monocyte (PBMC)-derived macrophages increased superoxide production. This effect did not require cell-cell contact. This increased superoxide was dependent on $\mathrm{PGE}_{2}$ activation of $\mathrm{PI} 3 \mathrm{~K}$, as antagonising $\mathrm{PI} 3 \mathrm{~K}$ or $\mathrm{PGE}_{2}$ production abrogated the effect, and was likely attributable to augmented recruitment of the NADPH oxidase catalytic complex to the phagosome. Similarly, in a rat model of Escherichia coli pneumonia, intratracheal administration of MSCs led to an increase in phagosomal superoxide in ex vivo isolated alveolar macrophages. Moreover, the investigators reported that PBMC-derived macrophages that had been co-cultured with MSCs showed both increased phagocytosis of un-opsonised E. coli or Burkholderia cenocepacia and increased bacterial killing. Importantly, this effect was also observed for macrophages derived from peripheral blood monocytes of septic patients.

Interestingly, in co-culture with MSCs, the monocyte-derived macrophages demonstrated at least two distinct cellular morphologies: one that was flattened, and another that was elongated, similar to polarised M2 macrophages. Fascinatingly, the increase in superoxide production (an M1-like behaviour) seemed confined to the flattened cells, while the more elongated cells rapidly acidified phagosomes and expressed CD163; features previously described as typical of M2 cells. The flattened, superoxide-producing MSCdifferentiated cells did not, however, express CD40, in contrast to bona fide M1 polarised macrophages, demonstrating that the oxidative MSC co-cultured macrophages could not be strictly called M1.

The studies by RABANi et al. [19] bring light to the seemingly conflicting prior data on the induction of some prototypically M1 behaviours with the suppression of others. In particular, they explored the dimorphism of monocyte-derived macrophages cultured with MSCs. M1 and M2 polarisation in vitro was previously thought to be somewhat mutually exclusive, since signalling intermediates of each polarisation pathway (particularly STAT1 and STAT6) inhibit one another in T-cells and are presumed to do the same in macrophages [20]. However, it is increasingly recognised that the complexity of the tissue milieu in vivo results in numerous macrophage phenotypes that are neither strictly M1 nor M2. In fact, MSC and other stromal cells in situ are likely to significantly influence these polarisation states, and co-culture in vitro may therefore be a step closer to recapitulating macrophage biology in vivo. Moreover, infections in vivo often lead to a mixture of type 1 and type 2 cytokines, which could lead to a mixture of M1-like and M2-like cells, or intermediate phenotypes. In fact, recent studies have shown that only some of the transcriptional outputs downstream of M1-polarising conditions are subject to inhibition by M2-inducing signals, and vice versa [21].

Several important questions stem from this work and will stimulate future investigations. First, are the antimicrobial effects of MSCs (either direct or through immune cells such as macrophages) observable with other types of bacteria, such as gram positives, intracellular, or encapsulated species, and with fungi, parasites and viruses? These pathogens contribute significantly to the pathogenesis of sepsis in some patients, and it will be important to understand whether patients who are septic from non-bacterial infections may benefit from MSCs. Several studies suggest benefit in viral infection [22-25], but results have been mixed. Second, the findings of dimorphic cultures of macrophages that appear neither truly M1 nor M2 begs for an unbiased investigation of the expression and behaviour of MSC co-cultured macrophages. Single cell sequencing, as the authors discuss, could be applied in this setting. Third, because myeloid cells in vivo are not a blank slate as they are in M0 cultures, it will be essential to determine how MSCs can influence the phenotype of macrophages that are subject to other physiologic or pathophysiologic signals. For instance, how will MSC co-culture affect cells that were previously polarised towards M2 in vitro. Fourth, how important is mitochondrial transfer from MSCs to macrophages for induction of these M1 and M2 phenotypes [26-28]?

Perhaps most importantly, further investigation into the influence of MSCs in vivo on tissue macrophages will be necessary. How will MSCs influence the phenotype of macrophages that are already previously differentiated and polarised in the tissue in vivo? Will this vary by tissue type, as some tissue macrophages 
tend towards a more M2-like phenotype at baseline, and others do not? In the setting of in vivo infection, do MSCs impart a similar dimorphic macrophage profile or does one phenotype dominate? And what is the time course of these effects? How might the dual phenotypes of MSC-influenced macrophages be of therapeutic benefit in other disease models, in addition to sepsis?

Perhaps one of the most promising features of MSCs is their ability to simultaneously exert antimicrobial and anti-inflammatory effects. This is in sharp contrast to heavy-handed immunosuppressive treatments that have shown harm, rather than benefit, to septic patients. Therefore, further understanding of the immunomodulatory properties of MSCs may be an essential step in developing targeted therapies for sepsis. One recent report showed that a single dose of intravenous MSCs appear to be safe in patients with sepsis [29], although there is much to be learned about which patients with sepsis and organ injury should be selected for cell-based therapy trials [30].

Conflict of interest: M.A. Matthay has received grants from NIH/NHLBI and FDA (for research/clinical trials), the Department of Defense (for clinical trials in ARDS), Bayer Pharmaceuticals (for an observational study of ARDS), GlaxoSmithKline (for an observational study of sepsis), Amgen (for animal studies of acute lung injury) and California Institute of Medicine (infrastructure, stem cell trials, UCSF), and has received personal fees from Roche-Genentec (for chairing DSMB of asthma trials), personal fees from CS Berling Inc, Cerus Therapeutics, Boerhinger-Ingelheim and Quark Pharmaceuticals (for consulting on ARDS), and personal fees from Thesan Pharmaceuticals (for consulting on lung pathology), outside the submitted work.

\section{References}

Gotts JE, Matthay MA. Sepsis: pathophysiology and clinical management. BMJ 2016; i1585.

Angus DC, van der Poll T. Severe sepsis and septic shock. N Engl J Med 2013; 369: 840-851.

Hotchkiss RS, et al. Sepsis and septic shock. Nat Rev Dis Prim 2016; 2: 16045.

4 Hotchkiss RS, Coopersmith CM, Mcdunn JE, et al. The sepsis seesaw tilting toward immunosuppression. 2009; 15: 496-497.

5 Iskander $\mathrm{KN}$, Osuchowski MF, Stearns-Kurosawa DJ, et al. Sepsis: multiple abnormalities, heterogeneous responses, and evolving understanding. Physiol Rev 2013; 93: 1247-1288.

6 Laroye C, Gibot S, Reppel L, et al. Concise review: mesenchymal stromal/stem cells: a new treatment for sepsis and septic shock? Stem Cells 2017; 35: 2331-2339.

7 Cheung TS, Dazzi F. Mesenchymal-myeloid interaction in the regulation of immunity. Semin Immunol 2018; 35: $59-68$.

8 Matthay MA, Pati S, Lee JW. Concise review: mesenchymal stem (stromal) cells: biology and preclinical evidence for therapeutic potential for organ dysfunction following trauma or sepsis. Stem Cells 2017; 35: 316-324.

9 Laffey JG, Matthay MA. Fifty years of research in ARDS. Cell-based therapy for acute respiratory distress syndrome. Biology and potential therapeutic value Am J Respir Crit Care Med 2017; 196: 266-273.

10 Kim J, Hematti P. Mesenchymal stem cell-educated macrophages: a novel type of alternatively activated macrophages. Exp Hematol 2009; 37: 1445-1453.

11 Selleri S, Bifsha P, Civini S, et al. Human mesenchymal stromal cell-secreted lactate induces M2- macrophage differentiation by metabolic reprogramming. Oncotarget 2016; 7: 30193-30210.

12 Chiossone L, Conte R, Spaggiari GM, et al. Mesenchymal stromal cells induce peculiar alternatively activated macrophages capable of dampening both innate and adaptive immune responses. Stem Cells 2016; 34: 1909-1921.

13 Asami, T. Ishii M, Namkoong H, et al. Anti-inflammatory roles of mesenchymal stromal cells during acute Streptococcus pneumoniae pulmonary infection in mice. Cytotherapy 2018; 20: 302-313.

14 Mei SHJ, Haitsma JJ, Dos Santos CC, et al. Mesenchymal stem cells reduce inflammation while enhancing bacterial clearance and improving survival in sepsis. Am J Respir Crit Care Med 2010; 182: 1047-1057.

15 Krasnodembskaya A, Samarani G, Song Y, et al. Human mesenchymal stem cells reduce mortality and bacteremia in gram-negative sepsis in mice in part by enhancing the phagocytic activity of blood monocytes. Am J Physiol Lung Cell Mol Physiol 2012; 302: L1003-L1013.

16 Krasnodembskaya, A, Song Y, Fang X, et al. Antibacterial effect of human mesenchymal stem cells is mediated in part from secretion of the antimicrobial peptide LL-37. Stem Cells 2010; 28: 2229-2238.

17 Murray PJ. Macrophage polarization. Annu Rev Physiol 2017; 79: 541-566.

18 Van Dyken SJ, Locksley RM. Interleukin-4- and interleukin-13-mediated alternatively activated macrophages: roles in homeostasis and disease. Annu Rev Immunol 2013; 31: 317-343.

19 Rabani R, Volchuk A, Jerkic M, et al. Mesenchymal stem cells enhance NOX2-dependent reactive oxygen species production and bacterial killing in macrophages during sepsis. Eur Respir J 2018; 51: 1702021.

20 Lawrence T, Natoli G. Transcriptional regulation of macrophage polarization: enabling diversity with identity. Nat Rev Immunol 2011; 11: 750-761.

21 Piccolo V, Curina A, Genua M, et al. Opposing macrophage polarization programs show extensive epigenomic and transcriptional cross-talk. Nat Immunol 2017; 18: 530-540.

22 Chan MCW, et al. Human mesenchymal stromal cells reduce influenza A H5N1-associated acute lung injury in vitro and in vivo. Proc Natl Acad Sci 2016; 113: 3621-3626.

23 Hui DS, Lee N, Chan PK, et al. The role of adjuvant immunomodulatory agents for treatment of severe influenza. Antiviral Res 2018; 150: 202-216.

24 Gotts JE, Abbott J, Matthay MA. Influenza causes prolonged disruption of the alveolar-capillary barrier in mice unresponsive to mesenchymal stem cell therapy. Am J Physiol Lung Cell Mol Physiol 2014; 307: L395-L406.

25 Zumla A, Rao M, Wallis RS, et al. Host-directed therapies for infectious diseases: current status, recent progress, and future prospects. Lancet Infect Dis 2016; 16: e47-e63. 
26 Islam MN, Das SR, Emin MT, et al. Mitochondrial transfer from bone-marrow-derived stromal cells to pulmonary alveoli protects against acute lung injury. Nat Med 2012; 18: 759-765.

27 Phinney DG, et al. Mesenchymal stem cells use extracellular vesicles to outsource mitophagy and shuttle microRNAs. Nat Commun 2015; 6: 8472 .

28 Jackson MV, Morrison TJ, Doherty DF, et al. Mitochondrial transfer via tunneling nanotubes is an important mechanism by which mesenchymal stem cells enhance macrophage phagocytosis in the in vitro and in vivo models of ARDS. Stem Cells 2016; 34: 2210-2223.

29 McIntyre L, et al. Cellular immunotherapy for septic shock (CISS): a phase I trial. Cytotherapy 2017; 19: e3-e4.

30 Gotts JE, Matthay MA. Cell-based therapy in sepsis. A step closer. Am J Respir Crit Care Med 2018; 197: 280-281. 